\title{
Effects of arsenite oxidation on metabolic pathways and the roles of the regulator AioR in Agrobacterium tumefaciens GW4
}

\author{
G.J. Wang, K.X. Shi, Q. Wang \& X. Fan \\ State Key Laboratory of Agricultural Microbiology, College of Life Science and Technology, \\ Huazhong Agricultural University, Wuhan, P.R. China
}

\begin{abstract}
To determine the related metabolic pathways mediated by As(III) oxidation and whether AioR regulates other cellular responses to As(III), proteomic and genetic analyses were performed on the heterotrophic arsenite-oxidizing Agrobacterium tumefaciens GW4. The results showed that AioR was the main regulator for As(III) oxidation, and As(III) oxidation altered several cellular processes, especially phosphate, cell wall and carbon metabolism. In addition, the synchronous regulation of As resistance/oxidation proteins and phosphate transport proteins indicated that the metabolism of As was highly associated with that of phosphate. AioR suppressed the expression of the pst2/pho2 system and up-regulated the pst1/pho1 system to transport As and phosphate economically. AioR is the key driver of strain GW4 to adapt well in the As(III)-enriched sediment environment.
\end{abstract}

\section{INTRODUCTION}

Agrobacterium tumefaciens GW4 is a highly As(III) resistant $[$ minimal inhibitory concentration $(\mathrm{MIC})=$ $25 \mathrm{mM}]$ and As(III)-oxidizing bacterium isolated from As-enriched groundwater sediments (Fan et al., 2008). Unlike most of the heterotrophic As(III)-oxidizing bacteria using As(III) oxidation as an As detoxification process, the As(III) oxidation of strain GW4 enhanced the bacterial growth, and the strain showed positive chemotaxis toward As(III) (Shi et al., 2017; Wang et al., 2015). However, the mutant strain GW4- $\Delta$ aioR failed to demonstrate increased growth, and its As(III) oxidation and As(III) chemotaxis phenotypes were both disrupted (Shi et al., 2017). It appeared that the effect of As(III) oxidation in strain GW4 was different from that in all the well-recognized heterotrophic and chemoautotrophic As(III)-oxidizing strains. We speculated that As(III) may be involved with several different metabolism pathways, and AioR may regulate other cellular functions besides As(III) oxidation.

Thus, in this study, we developed A. tumefaciens GW4 as a model to understand the alteration of global metabolism pathways with As(III) oxidation, and the regulatory roles of AioR. The methods includes isobaric tags for relative and absolute quantitation (iTRAQ) proteomics, gene knock out, and complementation and gene transcription analyses.

\section{MATERIALS AND METHODS}

\subsection{A. tumefaciens culture}

GW4 was grown at $28^{\circ} \mathrm{C}$ in $\mathrm{MMNH}_{4}$ medium containing $55 \mathrm{mM}$ mannitol as the primary carbon source and modified to contain $0.1 \mathrm{mM}$ phosphate (Liu et al.,
2012). Total protein was extracted from the control, the As(III) treated strain GW4 and GW4- $\Delta$ aioR cells. iTRAQ proteomics was analyzed using AB SCIEX nanoLC-MS/MS (Triple TOF 5600 plus).

\subsection{Gene knock out and complementation of aioR}

Gene knock out and complementation were performed as described in Wang et al. (2015) and Shi et al. (2017) and generated the mutant strain GW4- $\triangle a$ aioR and the complemented strain GW4- $\triangle$ aioR-C.

\subsection{Quantitative RT-PCR analysis}

Overnight cultures $\left(\mathrm{OD}_{600}=0.7-0.8\right)$ were inoculated into $100 \mathrm{~mL} \mathrm{MMNH}_{4}$ medium. RNA extraction and

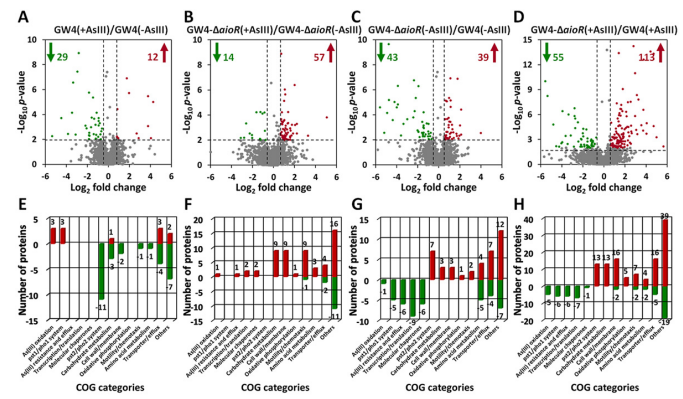

Figure 1. iTRAQ-based proteomic analysis of global perturbation of $A$. tumefaciens GW4 cellular metabolism in response to As(III) exposure. Gray vertical lines denote the 1.6 and -1.5 fold change designated cut-off. Horizontal gray lines denote a statistical significant $p$-value $\leq 0.01$. $(\mathrm{E}-\mathrm{H})$ Classification of proteins in COG categories. Red bars represent the up-regulated proteins and green bars represent the down-regulated proteins. 

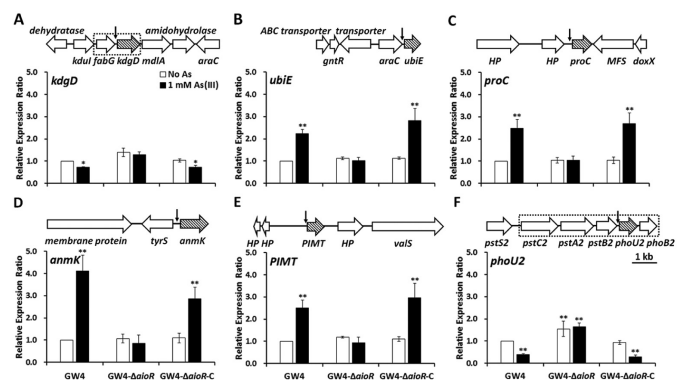

Figure 2. Influence of $\mathrm{As}(\mathrm{III})$ and AioR on the transcription of the $k d g D$ (A), ubiE (B), proC (C), anmK (D), PIMT (E) and phoU2 $(\mathrm{F})$ genes. The locations of the arrows are the AioR putative binding sites. The gene clusters in the dashed boxes are co-transcribed (data not shown).

Quantitative RT-PCR were performed as described before (Liu et al., 2012; Wang et al., 2015).

\section{RESULTS AND DISCUSSION}

\subsection{Proteiomics}

iTRAQ was compared in four treatments, GW4 (+AsIII) / GW4 (-AsIII), GW4- $\triangle$ aioR (+AsIII) / GW4- $\triangle$ aioR (-AsIII), GW4- $\triangle$ aioR (-AsIII) / GW4 (-AsIII) and GW4- $\triangle$ aioR (+AsIII) / GW4 (+AsIII). A total of 41, 71, 82 and 168 differentially expressed proteins were identified, respectively (Fig. 1).

\subsection{Quantitative RT-PCR analysis}

Gene transcription levels of the wild type GW4, GW4$\triangle$ aioR and GW4- $\triangle$ aioR-C showed that AioR affected the transcription of $k d g D, u b i E$, proC, anmK, PIMT and phoU2 genes (Fig. 2). EMSA analysis also showed that AioR interacted with the promoter region of these genes (data not shown). These genes are related to several metabolic pathways.

\subsection{The regulatory cellular networks controlled by AioR}

Based on our previous studies (Wang et al., 2015; Shi et al., 2017), and the above data, we suggested that the regulatory cellular networks controlled by AioR are As resistance (ars operon), phosphate metabolism (pst/pho system), TCA cycle, cell wall/membrane, amino acid metabolism and motility/ chemotaxis. AioR regulates several cellular processes. AioR regulates the expression of AioBA to oxidize $\mathrm{As}(\mathrm{III})$ (1.1), and regulates the expression of AioE to transfer the electron from AioBA to AioE with the generation of NADH (1.2). AioR could also regulate the expression of UbiE to maintain the electron transport within oxidative phosphorylation complex (1.2). AioR suppress the processes of glycolysis and TCA cycle by regulating $\mathrm{KdgD}$ (1.3). AioR also regulates the expression of Ars, AnmK, ProC and PIMT to participate in

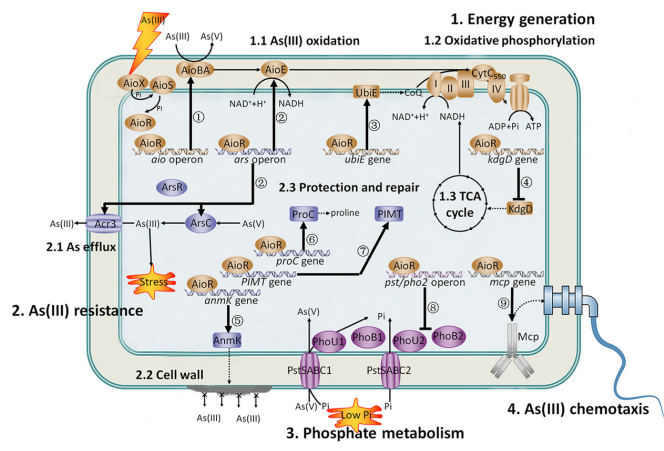

Figure 3. A general view showing the regulatory cellular networks controlled by AioR.

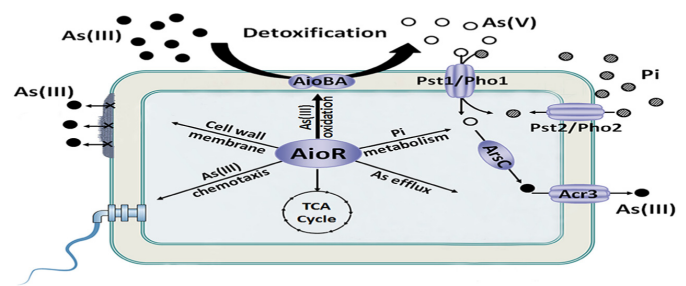

Figure 4. A general view showing of the roles of arsenic regulator AioR.

As efflux (2.1), recycling of peptidoglycan (2.2), synthesis of proline (2.3) and PIMT repair system (2.4) to enhance As(III) resistance. Besides, AioR regulates PhoU2-PhoB2 in phosphate metabolism and regulates Mcp in As(III) chemotaxis (Fig. 3).

In addition, the synchronous regulation of As resistance/oxidation proteins and phosphate transport proteins indicated that the metabolism of As was highly associated with that of phosphate. AioR suppressed the expression of the pst2/pho2 system and up-regulated the pst1/phol system to transport As and phosphate economically (Fig. 4).

\section{CONCLUSIONS}

A. tumefaciens GW4 represents a new type of heterotrophic As(III)-oxidizing bacteria that can use As(III) to enhance growth, and so which is different from most of the known heterotrophic As(III)oxidizing bacteria using As(III) oxidation as a detoxification process. Our results show that cellular responses to As(III) are delicate, and AioR mainly drives As(III) oxidation. Additionally, AioR could regulate multiple genes involved in phosphate metabolism, As(III) resistance, and energy acquisition. AioR suppressed the expression of the $p s t 2 / p h o 2$ system and up-regulated the pst1/phol system to transport As and phosphate economically. The regulatory cellular networks controlled by AioR provide the high As(III) resistance and 
better adaptation for strain GW4 to live in the As-rich sediment environment.

\section{ACKNOWLEDGEMENTS}

The present study was supported by the National Natural Science Foundation of China (31670108). During the revision of this abstract, a part of the results are accepted by Environmental Pollution (in press).

\section{REFERENCES}

Fan, H., Su, C., Wang, Y., Yao, J., Zhao, K., Wang, Y. \& Wang, G. 2008. Sedimentary arsenite-oxidizing and arsenatereducing bacteria associated with high arsenic groundwater from Shanyin, Northwestern China. J. Appl. Microbiol. 105: 529-539.
Liu, G., Liu, M., Kim, E.H., Maaty, W.S., Bothner, B., Lei, B., Rensing, C., Wang, G. \& McDermott, T.R. 2012. A periplasmic arsenite-binding protein involved in regulating arsenite oxidation. Environ. Microbiol. 14: 1624-1634.

Shi, K., Fan, X., Qiao, Z., Han, Y., McDermott, T.R., Wang, Q. \& Wang, G. 2017. Arsenite oxidation regulator AioR regulates bacterial chemotaxis towards arsenite in Agrobacterium tumefaciens GW4. Sci. Rep. 7: 43252.

Wang, Q., Qin, D., Zhang, S., Wang, L., Li, J., Rensing, C., McDermott, T.R. \& Wang, G. 2015. Fate of arsenate following arsenite oxidation in Agrobacterium tumefaciens GW4. Environ. Microbiol. 17: 1926-1940. 